\title{
Diagnostic utility of clinical and epidemiologic features in fever of unknown origin
}

\author{
Victor Roca Campañá ${ }^{\oplus}$, Rosa Eugenia Jiménez Paneque ${ }^{2} \odot$, Héctor Manuel Rodríguez Silva ${ }^{1}$ \\ ${ }^{1}$ Department of Internal Medicine, Clinical Surgical Hospital "Hermanos Ameijeiras". Havana, Cuba \\ ${ }^{2}$ Department of Nursing, Autonomous University of Chile, Faculty of Health Sciences, Santiago, Chile
}

DOI: $10.18621 /$ eurj.441463

\begin{abstract}
Objectives: To assess the diagnostic utility of clinical features in the major diagnostic categories of Fever of unknown origin (FUO).

Methods: One hundred and thirty-three patients meeting the classic criteria of FUO were included in the study. A structured diagnostic protocol was used in all cases. Sensitivity, specificity, positive and negative predictive values (PPV and NPVs), and likelihood ratios of positive and negative tests (LR+ and LR-) were estimated with $95 \%$ confidence intervals ( $95 \%$ CIs) for all clinical findings.

Results: Clinical and epidemiologic features with best diagnostic utility indexes for the three major diagnostic categories were: weight loss of 15 pounds or more (sensitivity, $68.4 \%, 95 \%$ CI: 52.33-84.52), pallor of the skin and mucous membranes (sensitivity, 65.7\%, 95\% CI: 49.39-82.19), prior medical history of cancer (PPV, 63.6\%, 95\% CI: 30.66-96.61; LR+, 4.38, 95\% CI: 1.36-14.09), lymphadenopathy (LR+, 2.2, 95\% CI: 1.114.74), for neoplasms; arthritis (PPV, 72\%, 95\% CI: 51.84-93.61), prior family history of collagen diseases (PPV, 100\%, 95\% CI: 91.67-100.00), neurologic disorder (LR+, 5.1, 95\% CI: 1.37-19.68), myalgia (LR+, 4.1, 95\% CI: 1.45-11.88) and skin lesions (LR+, 3.0, 95\% CI: 1.51-6.22) for noninfectious inflammatory diseases; weight loss of 15 pounds or more (sensitivity, 50\%,95\% CI: 27.91-72.09), epidemiological history of previous tuberculosis or tuberculosis exposure (LR+, 9.0, 95\% CI: 1.76-46.77), and jaundice (LR+, 2.73, 95\% CI: 0.710.63) for infections.

Conclusions: We identified clinical data emerging from the anamnesis and physical examination that may help to guide the diagnostic process in FUO.
\end{abstract}

Keywords: Fever of unknown origin, clinical features, diagnostic utility

Received: July 7, 2018; Accepted: July 4, 2019; Published Online: July 21, 2019

$\mathrm{F}$ ever of unknown origin (FUO) is a syndrome described more than 50 years ago. In 1961 Petersdorf and Beeson [1] defined FUO as an illness with temperature exceeding $38.3^{\circ} \mathrm{C}$ on at least three occasions, evolving during at least three weeks, with no diagnosis reached after one week of inpatient investigation.

Despite advances in diagnostic techniques, the di- agnostic process in patients with FUO remains a major clinical challenge. An enormous number of diseases and unusual presentations of common diseases have been reported as causes of FUO [2-4]. Clinicians are often unaware of the diagnostic significance of symptoms or physical findings in evaluating those patients. Besides, many symptoms are vague or seemingly insignificant [2].

Address for correspondence: Victor Roca Campañá, MD, PhD., Clinical Surgical Hospital "Hermanos Ameijeiras", Department of Internal Medicine, San Lázaro \# 701 esq. Belascoaín. Centro Habana, Ciudad de La Habana, Cuba. Código postal 10300. La Habana, Cuba

E-mail: victor.roca@infomed.sld.cu 
The value of finding clinical clues in the diagnostic process was accepted since the first FUO case series [5]. In 1997 De Kleijn et al. [6] gave much weight to the presence of potentially diagnostic clues (PDCs) in history and physical examination even when many of them were misleading in their report. Gaeta, Vanderschueren and Knockaert also highlighted the importance of PDC to reach a diagnosis in FUO patients $[7,8]$.

On the other hand, the body of FUO literature consists of case series and cohort studies and the yield from a complete history review and meticulous physical examination is not well known [9-11]. This has resulted in excessive diagnostic testing because there is not an efficient and effective diagnostic approach $[3,9]$.

Authors like Cunha [3,12] and Tolia [2] have emphasized the value of history and physical examination to identify the cause of FUO. The knowledge of clinical aspects in FUO patients will lead physicians to be focused on what to look for and its diagnostic significance [10]. Diagnostic approach in FUO requires considering clinical presentation that can group patients in specific categories: infectious, noninfectious inflammatory diseases (NIID), neoplasms, and miscellaneous disorders, as has been suggested by Cunha $[10,12]$.

Herein we present a prospective 10-year study on 133 patients presenting with FUO to a tertiary care center. Our objective was to estimate the diagnostic utility of clinical and epidemiological features to recognize whether the disease is infectious, noninfectious inflammatory or neoplastic.

\section{METHODS}

Between January 2000 and December 2009, we recruited consecutive patients with FUO at the "Hermanos Ameijeiras" Hospital in Havana, Cuba. This is a 644-bed hospital facility for adults with 14 clinical and 12 surgical wards. All the patients included in this study were admitted in one of the four Internal Medicine wards existing now in this tertiary care center. The study was approved by the hospital Research Ethics Committee.

\section{Eligibility Criteria}

Patients had to meet the classic criteria of FUO for being included in the study: febrile illness persisting for three weeks or more, fever of more than $38.3^{\circ} \mathrm{C}$ on at least three occasions and an unclear diagnosis after one week of hospital investigation. Patients were excluded if they were known to have HIV infection or if they did not present fever during their hospitalization. All patients were older than 16 years.

\section{Diagnostic Work-up}

Since 1998 our hospital has a structured diagnostic FUO protocol. Data registered include history, physical examination and investigations required in the diagnostic process. All this information has been prospectively registered in a structured data collection form. This protocol was not applied in a rigid manner in all patients.

The protocol consists in one week of hospitalization for cases with fever lasting three weeks or more. During the first week of hospitalization patients undergo a standardized history and standardized thorough physical examination. Fever existence is confirmed during this week and all medications are withdrawn if possible.

Investigations are categorized as obligatory tests, first-level tests and second-level tests. Obligatory tests are part of the initial diagnostic evaluation of every patient, they must be performed in all patients in the first week of admission and are required to qualify as FUO. These tests include a battery of nonspecific laboratory tests, basic imaging tests and blood cultures. First-level tests and second-level tests are done in cases who still meet FUO criteria after this first week. First-level tests include investigations with more complexity without biopsy procedures, such as: immunological tests, serological tests, microbiological tests, imaging techniques, endoscopic procedures, and endocrinological tests. Second-level tests comprise invasive procedures (Table 1).

Clinical presentation and results of obligatory investigations were considered to determine if first or second-level tests should be indicated. If a patient remains without definite diagnosis after first-level tests results, second-level tests are to be considered. Liver biopsy was considered only in patients with 
Table 1. Summary of tests in the diagnostic protocol

\section{Obligatory tests performed in all patients}

Hemoglobin; leukocyte count and differential count; platelet count; sedimentation rate; creatinine; aspartate aminotransferase, alanine aminotransferase, alkaline phosphatase, blood cultures $(\mathrm{n}=3)$, urinalysis (microscopic examination), chest X-ray, abdominal ultrasonography of abdomen.

First-level tests: immunological tests, microbiological tests, serological tests, imaging techniques, endoscopic procedures, endocrinological tests

Lactate dehydrogenase; protein; protein fractions; ferritin; creatine phosphokinase; bilirubin; ANA; anti-DNA; rheumatoid factors; ANCA; anticardiolipin antibodies; serum protein electrophoresis; cryoglobulin; $\mathrm{C} 3$ and $\mathrm{C} 4$ levels; blood cultures; urine cultures; HBsAg; anti-HCV; HIV serology; Western blot test; serology for citomegalovirus, Epstein-Barr virus, Brucella serologic tests; tuberculin skin test; AFB smear and culture of sputum and effusions (pleural, peritoneum, pericardium); ultrasound searching lymphadenopathy; complete lower extremity ultrasound; abdominal CT; chest CT; transthoracic echocardiography; transesophageal echocardiography, colonoscopy, gastroscopy, bronchoscopy, thyroid function tests (TSH, T4).

\section{Second-level tests: invasive procedures}

Bone marrow aspiration and biopsy; liver biopsy; lymph node dissection; kidney biopsy; skin and muscle biopsies; solid tumors biopsy; temporal artery biopsy, aspiration of pleural fluid or ascites, pleural biopsy, peritoneum biopsy, exploratory laparotomy.

$\mathrm{ANA}=$ antinuclear antibodies, anti-DNA $=$ anti-native $\mathrm{DNA}$ antibody, $\mathrm{ANCA}=$ antineutrophil cytoplasmic antibody, HBsAg = hepatitis $\mathrm{B}$ surface antigen, anti-HCV=hepatitis $\mathrm{C}$ virus antibodies, HIV=human immunodeficiency virus, $\mathrm{AFB}=$ acid-fast bacilli, $\mathrm{CT}=$ computed tomography

abnormal liver function test.

In selected cases without definite diagnosis empirical therapy was required according to their clinical condition and to their presumptive diagnosis. Such empiric therapy included antimicrobial agents, anti-tuberculous drugs or corticosteroids.

For our study, during hospitalization all patients were assessed weekly by the first author besides the attending physician. The final diagnosis was established by the attending physician and two members of the FUO Study Group of the hospital (including the first author). The conclusive factors in the establishment of the final diagnosis included diagnostic test results or classification criteria. In some patients, final diagnosis was made based on their response to specific therapy or based on their clinical course with exclusion of other diseases. In each patient the final diagnosis was grouped in four separate diagnostic categories: infections, noninfectious inflammatory diseases, neoplasms or miscellaneous category. When the final diagnosis did not fit into one of the three major FUO groups it was considered a miscellaneous category.

The patients discharged without final diagnosis were followed until a diagnosis could be made or for one and a half year. This follow-up was performed by the attending physician and the first author at our institution.

Diagnostic tests and clinical criteria followed to establish definitive diagnosis and to place the patients in one of the major diagnostic categories of FUO are detailed in Table 2. The miscellaneous group and patients without final diagnosis are not included.

The presence or absence of potentially relevant findings in the clinical history and physical examination for approaching the diagnosis of FUO, were registered as:

Prior medical history: neoplastic disorders, collagen diseases, history of heart murmur, history of surgical-invasive procedures during the last six months before the onset of fever, history of dental work or periodontal disease during the last three months before the onset of fever, history of blood transfusion, history of glucocorticoids or immunosuppressive treatment during the last three months before the onset of fever.

Prior family illnesses (parents, brothers, children, grandparents, uncle): neoplastic disorders, collagen 
Table 2. Tests for definitive diagnosis in major diagnostic categories of Fever of unknown origin

\begin{tabular}{|c|c|c|c|c|c|c|}
\hline \multirow{2}{*}{$\begin{array}{l}\text { Diagnostic } \\
\text { categories }\end{array}$} & \multicolumn{6}{|c|}{ Definitive diagnostic tests } \\
\hline & Histology & Inmunology & Imaging & Microbiology & Laboratory & Clinical \\
\hline Neoplasms & $\begin{array}{l}\text { bone marrow biopsy, } \\
\text { lymphadenopathy } \\
\text { biopsy, } \\
\text { lymphadenopathy } \\
\text { cytology, solid tumors } \\
\text { biopsy, post-mortem } \\
\text { examination }\end{array}$ & & & & & \\
\hline $\begin{array}{l}\text { Noninfectious } \\
\text { inflammatory } \\
\text { diseases }\end{array}$ & $\begin{array}{l}\text { muscle biopsy, skin } \\
\text { biopsy, renal biopsy }\end{array}$ & $\begin{array}{l}\text { ANA, anti-DNA, } \\
\text { RF, ANCA, } \\
\text { anticardiolipin } \\
\text { antibodies }\end{array}$ & $\begin{array}{l}\text { Axial contrast } \\
\text { enhanced CT of } \\
\text { supra-aortic trunks }\end{array}$ & & $\begin{array}{l}* \dagger \text { ESR } \\
>50 \mathrm{~mm} / \mathrm{h}, \\
\ddagger \text { leukocytosis } \\
>10.000 \text { (>80\% } \\
\text { granulocytes), } \\
\text { †liver function } \\
\text { tests, serum } \\
\text { †ferritin }\end{array}$ & $\begin{array}{l}* \text { Age }(\geq 50 \\
\text { years), headache, } \\
\text { visual symptoms, } \\
\text { jaw claudication. } \\
\dagger \text { Aching and } \\
\text { stiffness of } \\
\text { shoulders and } \\
\text { hips. } \\
\text { †Arthralgia, } \\
\text { arthritis, fever } \\
>39^{0} \mathrm{C}, \text { transient } \\
\text { erythema, sore } \\
\text { throat, } \\
\text { generalized } \\
\text { lymphadenopathy } \\
\text { splenomegaly. } \\
* \dagger+\text { Response to } \\
\text { corticosteroid } \\
\text { therapy. } \\
*+\dagger \text { Exclusion of } \\
\text { infections, } \\
\text { malignancies and } \\
\text { rheumatic } \\
\text { diseases }\end{array}$ \\
\hline Infections & $\begin{array}{l}\text { lymphadenopathy } \\
\text { biopsy, liver biopsy, } \\
\text { bone marrow biopsy, } \\
\text { post-mortem } \\
\text { examination }\end{array}$ & & $\begin{array}{l}\text { abdominal } \\
\text { ultrasonography, } \\
\text { abdominal CT, } \\
\text { transthoracic } \\
\text { echocardiography, } \\
\text { transesophageal } \\
\text { echocardiography }\end{array}$ & $\begin{array}{l}\text { urine culture, } \\
\text { pleural fluid } \\
\text { cultures AFB } \\
\text { positive, } \\
\text { HBsAg, } \\
\text { Brucellosis } \\
\text { serologic tests, } \\
\text { HIV serology, } \\
\text { Western blot } \\
\text { test }\end{array}$ & & $\begin{array}{l}\text { clinical course, } \\
\text { response to } \\
\text { antibiotic } \\
\text { therapy, response } \\
\text { to antituberculous } \\
\text { therapy }\end{array}$ \\
\hline
\end{tabular}

$\overline{\mathrm{HIV}}=$ human immunodeficiency virus, ANA $=$ antinuclear antibodies, anti-DNA $=$ anti-native DNA antibody, ANCA $=$ antineutrophil cytoplasmic antibody, $\mathrm{RF}=$ rheumatoid factor, $\mathrm{CT}=$ computed tomography, $\mathrm{AFB}=$ acid-fast bacilli, $\mathrm{HBsAg}=$ hepatitis $\mathrm{B}$ surface antigen, $\mathrm{ESR}=$ erythrocyte sedimentation rate.

Noninfectious inflammatory diseases: Clinical and laboratory criteria were followed to establish the diagnosis of * giant cell arteritis, $\dagger$ polymyalgia rheumatic and †adult-onset Still disease. 
diseases.

Prior epidemiological history: previous tuberculosis or tuberculosis exposure, previous cattle or bird exposure, travel history.

History: recurrent fever (a fluctuating fever pattern with fever-free intervals of at least two weeks), duration of fever longer than six months, night sweats, chills, myalgia, arthralgia, visual complaints (amaurosis fugax, decreased visual acuity, blurred vision), headaches, abdominal pain, new onset of back pain, chest pain, diarrhea, shortness of breath, dry cough, sputum, dysuria, dysphagia, lower gastrointestinal bleeding.

Physical findings: pallor of the skin or mucous membranes, weight loss of 15 pounds or more, mouth ulcers, heart murmur, hepatomegaly, splenomegaly, localized or generalized lymphadenopathy, abdominal tumor, arthritis, temporal artery tenderness, skin lesions (erythema, macule, papule, ulcers, purpura, necrosis), tachycardia, pulmonary auscultation abnormalities (crackles, rhonchi, wheezes, absent breath sounds), jaundice, diminished or absent pulse, neurologic disorder (seizures, mental status changes, cranial nerve palsies, peripheral neuropathy, sensory loss, motor deficit, neuropsychiatric manifestations with cognitive dysfunction).

\section{Statistical Analysis}

Sensitivity, specificity, positive predictive value (PPV), negative predictive value (NPV), likelihood ratio of a positive test and likelihood ratio of a negative test (LR+ and LR-) were estimated (with 95\% confidence intervals [95\% CI]) for each of the clinical and epidemiological features to assess their contribution to the diagnosis of the major etiologic categories of FUO: infectious, noninfectious inflammatory diseases and neoplastic disorders. The gold standard was the definitive diagnosis at the end of the clinical workup. We performed Statistical analysis with SPSS v 13.0, and Epidat 3.1 (Xunta de Galicia, Conselleria de Sanidade, Direccion Xeral de Saúde Pública, https://dxsp.sergas.es ).

We considered a clinical or an epidemiological feature useful for diagnosis if at least one of the following criteria were met: sensitivity higher or equal to $50 \%$, likelihood ratio of a positive test higher or equal to 2, positive predictive value superior to $60 \%$, in one or more FUO groups.

\section{RESULTS}

Between January 2000 and December 2009, 150 adult patients were admitted to our internal medicine wards with prior diagnosis of FUO. Of these patients, 133 were eligible for inclusion and were retained for analysis; 17 cases were excluded (in nine patients, diagnosis was achieved during the first week of hospital investigation, and in eight patients, temperature over $38.30 \mathrm{C}$ could not be documented during hospitalization).

The mean age of our cases was $50.4 \pm 18$ years (range, 16 to 91 years). Fifty-two patients (39.1\%) were elderly (over 60 years old). Sixty-five patients $(48.9 \%)$ were male. The mean duration of hospitalization to establish definitive diagnosis was $28.1 \pm 20.9$ days (range, 5 to 149 days). Before admission to our institution, sixty-five patients $(49.6 \%)$ were hospitalized at least once in general Internal Medicine wards and fifty patients $(37.6 \%)$ were referred by internists of other university hospitals.

The most prevalent diagnostic categories of FUO were: 38 neoplasms $(28.6 \%)$ and 37 noninfectious inflammatory diseases $(27.8 \%)$ followed by 24 infections (18\%), 18 miscellaneous diseases (13.5\%), and 16 undiagnosed patients (12\%). Malignant lymphoma, adult-onset Still disease and abdominal abscess were the most common causative diseases in each FUO group respectively (Table 3 ).

Patients in whom no final diagnosis was made were followed-up for 18 months, 14 patients recovered spontaneously, and in two patients fever subsided after empirical treatment with corticosteroids.

In six patients, diagnosis could be achieved by autopsy: two non-Hodgkin lymphomas, one adrenocortical carcinoma, one bone marrow toxicity, one miliary tuberculosis, and one bacterial liver abscess.

\section{Diagnostic utility for clinical history and physical examination}

In the neoplasms category, the sensitivity of 
Table 3. Final diagnosis in 133 patients with Fever of unknown origin

\begin{tabular}{|c|c|c|}
\hline & Diagnostic category & No. of patients (\%) \\
\hline \multirow[t]{6}{*}{ Infections } & & $24(18)$ \\
\hline & Bacterial & \\
\hline & Abdominal abscess & 5 \\
\hline & Endocarditis & 4 \\
\hline & Tuberculosis & 4 \\
\hline & Other * & \\
\hline \multicolumn{3}{|l|}{ Viral } \\
\hline & HIV infection & 4 \\
\hline & Chronic hepatitis B or C & 3 \\
\hline Neoplasms & & $38(28.6)$ \\
\hline \multicolumn{2}{|c|}{ Haematological } & 24 \\
\hline & Lymphoma & 19 \\
\hline & Chronic myeloproliferative disorder & 3 \\
\hline & Other ${ }^{+}$ & \\
\hline \multicolumn{2}{|c|}{ Solid tumours } & 14 \\
\hline & Metastasis of unknown origin & 4 \\
\hline \multicolumn{3}{|c|}{ Other $\$$} \\
\hline \multicolumn{2}{|c|}{ Non-infectious inflammatory diseases } & $37(27.8)$ \\
\hline \multicolumn{2}{|c|}{ Connective tissue diseases } & 21 \\
\hline \multicolumn{2}{|c|}{ Adult Still's disease } & 10 \\
\hline \multicolumn{2}{|r|}{ Systemic lupus erythematosus } & 6 \\
\hline \multicolumn{2}{|r|}{ Rheumatoid arthritis } & 3 \\
\hline \multicolumn{3}{|c|}{ Other $\S$} \\
\hline \multicolumn{2}{|c|}{ Vasculitis syndromes } & 15 \\
\hline \multicolumn{2}{|c|}{$\begin{array}{l}\text { Temporal arteritis/Polymyalgia } \\
\text { rheumatic }\end{array}$} & 7 \\
\hline \multicolumn{2}{|r|}{ Polyarteritis nodosa } & 4 \\
\hline \multicolumn{2}{|r|}{ Wegener's granulomatosis } & 2 \\
\hline \multicolumn{2}{|r|}{ Other $\|$} & \\
\hline \multicolumn{2}{|l|}{ Miscellaneous } & $18(13.5)$ \\
\hline & Factitious fever & 3 \\
\hline & Deep vein thrombosis & 2 \\
\hline & 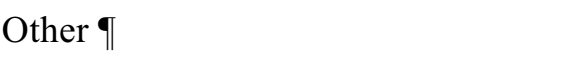 & \\
\hline \multicolumn{2}{|l|}{ No diagnosis } & $16(12)$ \\
\hline
\end{tabular}

* includes Brucellosis 2 cases, urinary tract infection 1 case, biliary tract infection 1 case. $\dagger$ includes multiple myeloma 1 case, acute myelogenous leukemia 1 case.

$\mp$ includes solid malignant tumors: lung 2 cases, colon 1 case, prostatic 1 case, bladder 1 case, adrenal gland 1 case, thyroid 1 case, uterus 1 case, mediastinal angiosarcoma 1 case, gastric GIST 1 case.

$\S$ includes polymyositis 1 case, dermatomyositis 1 case.

\| includes Takayasu's arteritis 1 case, microscopic polyangiitis 1 case, anti-phospholipid syndrome 1 case.

I includes Castleman's disease 1 case, Kikuchi's disease 1 case, sarcoidosis 1 case, inflammatory pseudotumor 1 case, nonhematologic bone marrow fibrosis 1 case, autoimmune hepatitis 1 case, chronic meningitis 1 case, idiopathic granulomatosis 1 case, aortic dissection 1 case, iliac artery aneurysm 1 case, common variable immunodeficiency 1 case, drug-induced liver injury 1 case, interstitial pneumonia 1 case. 
weight loss of 15 pounds or more $(68.4 \%, 95 \% \mathrm{CI}$ : 52.33 - 84.52) and pallor of skin or mucous membranes $(65.7 \%, 95 \%$ CI: $49.39-82.19)$ is noticeable. Almost 64\% (95\% CI: 30.66-96.61) of patients with prior medical history of neoplastic disorder had cancer as the cause of FUO (positive predictive value). Other clinical features showed low sensitivity, but specificity was over $90 \%$ for medical history of neoplastic disorder, medical history of immunosuppressive treatment and jaundice. Only one clinical aspect exhibited a fair likelihood ratio of a positive test: medical history of neoplastic disorder (LR+=4.38, 95\% CI: 1.36-14.09).

In noninfectious inflammatory diseases category: pallor of skin or mucous membranes showed the higher sensitivity $(56.76 \%, 95 \%$ CI: 39.44-74.07). Noticeable positive predictive values were present for visual complaints (75\%, 95\% CI: 20.07-100.00), arthritis (72.73\%, 95\% CI: 51.84-93.61), mouth ulcers (70\%, 95\% CI: 36.60-100) and prior family illnesses of collagen diseases (100\%, 95\% CI: 91.67-100.00). Clinical features as visual complaints, mouth ulcers, arthritis, neurologic disorder, diminished or absent pulse, myalgia, arthralgia, skin lesions and headaches were found between eight and three times more often in patients of the EINI group than in patients of other FUO groups $(\mathrm{LR}+)$ but only arthritis showed an important confidence interval $(\mathrm{LR}+=6.92,95 \% \mathrm{CI}$ : 2.93-16.32). For this group of patients, specificity was very high for all features listed in Table 4 except pallor of the skin or mucous membranes.

In infectious diseases category, likelihood ratio of a positive result was fairly high for previous tuberculosis or tuberculosis exposure (LR $+=9.08$, 95\% CI: 1.76-46.77). Sixty-six percent of patients with epidemiological history of previous tuberculosis or tuberculosis exposure had an infectious disease as cause of FUO (PPV, 95\% CI: 20.61-100\%). Specificity was high for previous tuberculosis or tuberculosis exposure (98.17, 95\% CI: 95.19-100) and jaundice (95.41, 95\% CI: 91.03-99.80).

In Table 4 we display the diagnostic utility indexes of clinical history and physical exam features considered useful (see the methods section) for each of the three main diagnostic categories.

We found some other interesting and more specific clinical features for which no diagnostic utility indexes were estimated since they were present in very few cases, but we still consider them worthy of exposing. In the neoplasms group, the period between prior history of malignancy and FUO ranged from two to six years. Twenty-one percent of patients with malignant lymphoma had recurrent fever. Four patients with jaundice had a hematologic malignancy, two had a malignant lymphoma, one had acute myeloid leukemia, and one had a chronic myeloproliferative disorder. Lymphadenopathy was localized in eight patients and generalized in three patients in this group of FUO: five were cervical, three were inguinal, two were, supraclavicular, and one was axillary.

Regarding the noninfectious inflammatory diseases group, arthritis occurred mainly in adult-onset Still disease and in systemic lupus erythematosus (five patients each). Adult-onset Still disease was the main cause of skin lesions, sometimes rash persisted for a few days. Recurrent fever pattern was not present in patients with this inflammatory disorder.

Visual complaints in systemic inflammatory diseases were amaurosis fugax (Takayasu's arteritis) and blurred vision or decreased visual acuity (giant cell arteritis, adult-onset Still disease).

Neurologic disorders present in systemic inflammatory diseases were: peripheral mononeuropathy (polyarteritis nodosa) in two patients, diplopia due to the sixth cranial nerve palsy (one giant cell arteritis, and one adult-onset Still disease), hemiparesis (one patient with secondary antiphospholipid syndrome), and neuropsychiatric manifestations in one patient with lupus erythematosus.

In the infectious diseases group, chronic hepatitis B (three patients) and AIDS (three patients) were the most common etiology for weight loss of 15 pounds or more. The most common causes of jaundice in this group were pyogenic liver abscess, chronic hepatitis $\mathrm{B}$, and AIDS (one patient each).

\section{DISCUSSION}

In this prospectively collected series of FUO, we attempted to assess the utility of the clinical aspects in the categories of neoplasms, noninfectious 
Table 4. Epidemiologic and clinical features with diagnostic utility* in major diagnostic categories

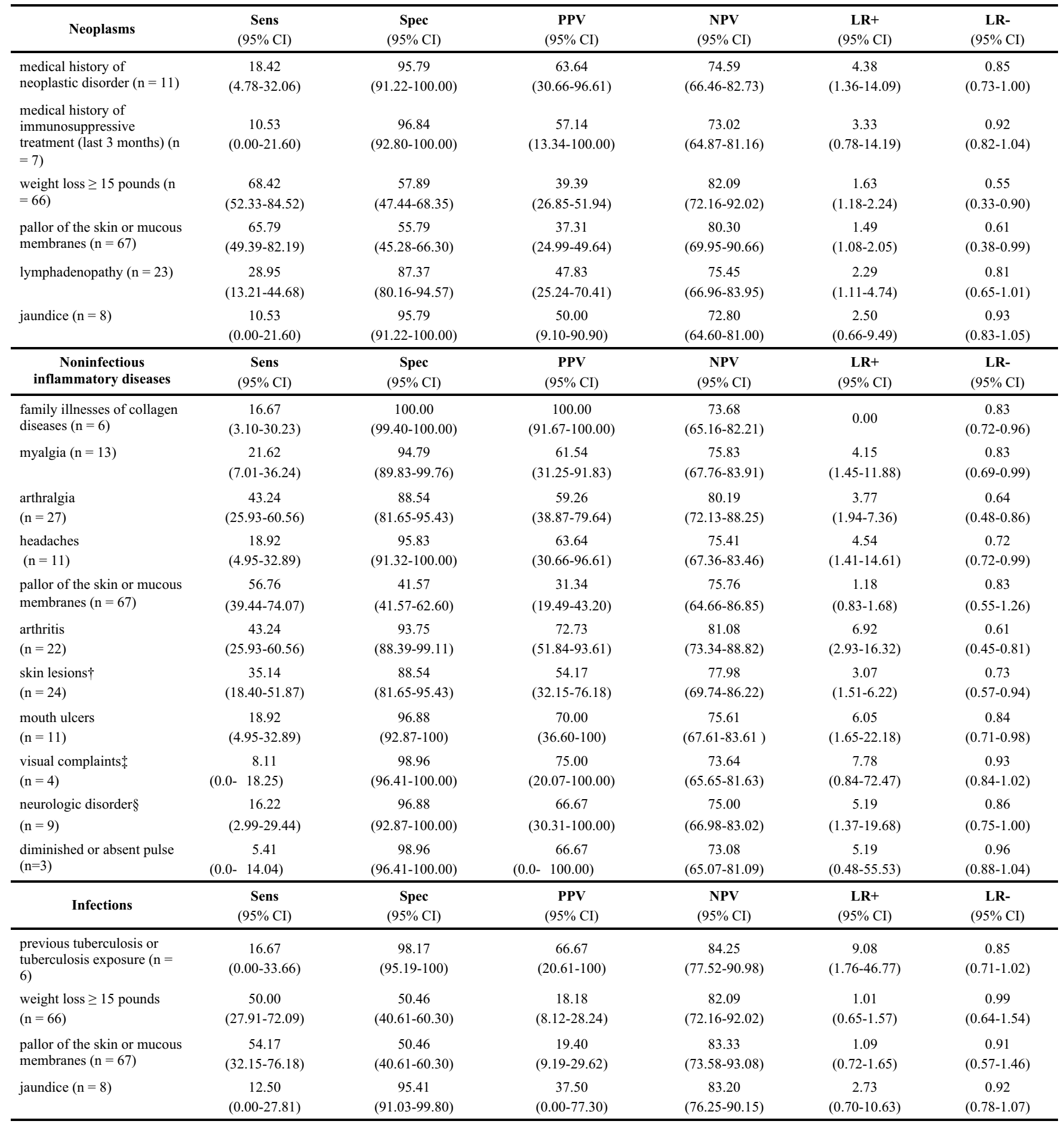

Sens $=$ sensitivity, Spec $=$ specificity, PPV $=$ positive predictive value, NPV $=$ negative predictive value, $\mathrm{LR}+=$ likelihood ratio of a positive test, LR- $=$ likelihood ratio of a negative test.

* Diagnostic utility = All clinical or epidemiological aspects meeting at least one of the following criteria: sensitivity higher or equal to $50 \%$, likelihood ratio of a positive test higher or equal to 2 , positive predictive value superior to $60 \%$,in one or more FUO groups.

$\dagger$ includes erythema, macule, papule, ulcers, purpura, and necrosis.

$\$$ includes amaurosis fugax, decreased visual acuity, blurred vision.

§includes seizures, mental status changes, cranial nerve palsies, peripheral neuropathy, sensory loss, motor deficit, neuropsychiatric manifestations with cognitive dysfunction.

|| Diagnostic value indexes for all features are available from the author upon request. 
inflammatory diseases and infections. We used the common diagnostic utility indexes for their summarizing capacity and acceptance. We also described some features present in a reduced number of patients, worthy of a mention. FUO is a complex syndrome always difficult to etiologically diagnose and thus a challenge for clinicians worldwide $[3,10]$. It is also an infrequent syndrome, our series is not a small one, considering other published series, and perhaps one of the few case series paying attention to the epidemiological and clinical history of the patient $[5,11,13-15]$.

According to a previous report, the prevalence of FUO groups in our center has changed during the past two decades [16]. Neoplasms (40\%) and infections (29.5\%) frequency has declined but neoplasms still represent the main diagnostic category [16]. The prevalence of NIID in the present series was higher than in the previous report (16.1\%) [16]. This trend could be the result of: 1) improvements in diagnostic methods for certain entities (endocarditis, abdominal abscesses, tumors), 2) referral patterns to our tertiary care center, 3) diagnosis of some NIID (Still disease, vasculitis) is rarely obtained at an early stage, 4) hematologic malignant diseases and small metastasis remain difficult to diagnose.

The large mean duration of hospitalization in this series revealed the major difficulty in establishing the cause of FUO. Other authors report similar values for this variable with periods ranging from 21 to 27 days $[13,14,17]$.

De Kleijn et al. [13] and Iikuni et al. [15] found common clinical findings in FUO patients such as: relevant diseases in the past $(78.4 \%)$, weight loss $(55.7 \%)$, lymphadenopathy $(41.2 \%)$, arthralgia $(28.7 \%)$, skin rash $(16.3 \%)$, hepatosplenomegaly (14.4\%), and heart murmur (12.4\%). Some of these clinical findings were also frequently observed in this report.

These report findings (high positive predictive value and high likelihood ratio of a positive test) led to consider the neoplastic etiology in patients with prior history of cancer, no matter how remote. In such cases we found spread of the tumor or its transformation in cases with prior hematological malignant diseases. Similarly, other authors [2] recognize the value of this diagnostic clue and De
Kleijn et al. [13] suggest to always search for recurrence of the tumor in these cases.

The patients who receive immunosuppressive treatment may become neutropenic and the fever will be due to infections in most of such cases [18, 19]. However, in this series the patients with prior history of immunosuppressive treatment were not neutropenic, and an infection was not the cause of their fever.

Based on the current study results a weight loss of 15 pounds or more pointed in first place to the neoplastic category and in second place to the infectious group. Hirschmann et al. [20] and Cunha et al. $[10,12]$ reported that weight loss accompanied by dramatic loss of appetite suggests a neoplastic etiology. Bleeker-Rovers et al. [14] considered weight loss as a clue with diagnostic value in FUO patients. It is well known that weight loss can be a remarkable clinical finding in common neoplastic or infectious causes of FUO such as malignant lymphomas, tuberculosis, subacute bacterial endocarditis and HIV infection [10, 21-27].

In this study, palpable lymphadenopathy mainly pointed to the neoplastic group. Clinical lymphadenopathy can be found in non-neoplastic diseases but since 1982 and thereafter some authors have considered this physical finding as a helpful clue to diagnose neoplasms, particularly malignant lymphomas [5, 10, 11, 28, 29]. Unlike other reports $[3,6,30]$, in this series lymphadenopathy confined to the inguinal region was also useful in establishing a definitive diagnosis.

The high positive predictive value and the high likelihood ratio of a positive test of some clinical findings in the NIID category confirmed them as clues with diagnostic utility in this group. Similar observations have been indicated by Cunha et al. [3, $10,12]$ who stated the family medical history of rheumatic disorders, eye symptoms, joint swelling and effusion, myalgia, arthralgia, mouth ulcers and headache as helpful clues in this FUO category. In addition, Iikuni et al. [15] reported arthralgia (42.2\%), skin rash $(31.1 \%)$, and myalgia (13.3\%) as relevant findings in this category.

Our observations also confirm that the presence of certain visual complaints (amaurosis fugax, decreased visual acuity, blurred vision) or neurologic disorders 
(cranial nerve palsies, peripheral mononeuropathy, motor deficit, neuropsychiatric manifestations) pointed strongly to the diagnosis of vasculitis syndromes. In agreement with this result, some authors $[3,10,11,31]$ have described these clinical findings mainly in temporal arteritis, Takayasu's arteritis, periarteritis nodosa and systemic lupus erythematosus.

Tuberculosis presenting as FUO can be difficult to diagnose due to commonly nonspecific signs and symptoms $[25,32,33]$. This study found high positive predictive value and high likelihood ratio of a positive test for previous tuberculosis or tuberculosis exposure in the infectious category. The epidemiological value of these antecedents has been recognized in other reviews [10].

We found jaundice associated with involvement of the liver by non-TB infections or neoplasms. Prior studies do not describe jaundice in FUO patients $[2,3,13-15]$. Its presence has been related with biliary obstruction by tuberculous lymphadenitis [32].

Pallor of the skin or mucous membranes showed a high sensitivity in all of the FUO groups evaluated. This result is in accordance with the statement that low hemoglobin increases the chance of reaching a diagnosis in FUO patients [6, 34].

Our results confirmed that recurrent fever and fever lasting longer than six months were unhelpful clues. De Kleijn et al. [13] and Knockaert et al. [35] have associated these clinical findings with low probability to make a diagnosis.

\section{Limitations}

Finally, we point out some limitations of our study. The first and foremost is the relatively low number of cases that prevented accurate estimates of the diagnostic indicators. The fact that the study is based on information from real patients in whom the indications of diagnostic procedures depend on the patient and his treating physician, is the second important limitation of the study since it might bias the diagnostic value indicators. Although we have a diagnostic protocol for FUO, due to ethical considerations total compliance is not possible to achieve in all patients.

The study was conducted in a tertiary care center, so extrapolations to primary or secondary care facilities should be done with caution.

\section{CONCLUSION}

In conclusion, the present study reveals clinical and epidemiological features with diagnostic utility in the major diagnostic categories of FUO and quantifies this utility.

\section{Authors Contribution}

VRC $=$ Study concept and design, data collection and interpretation, drafted and revised the article; RSJP $=$ Design of the study and statistical planning, data analysis and interpretation; and HMRS = Study concept and design, data interpretation. All authors participated in the critical revision of the article and approved the final version to be published.

\section{Conflict of interest}

The author disclosed no conflict of interest during the preparation or publication of this manuscript.

\section{Financing}

The author disclosed that they did not receive any grant during conduction or writing of this study.

\section{REFERENCES}

[1] Petersdorf RG, Beeson PB. Fever of unexplained origin: report on 100 cases. Medicine (Baltimore) 1961;40:1-30.

[2] Tolia J, Smith LG. Fever of unknown origin: historical and physical clues to making the diagnosis. Infect Dis Clin North Am 2007;21:917-36.

[3] Cunha BA. Fever of unknown origin: focused diagnostic approach based on clinical clues from the history, physical examination, and laboratory tests. Infect Dis Clin North Am 2007;21:1137-87.

[4] Mulders-Manders CM, Simon A, Bleeker-Rovers CP. Rheumatologic diseases as the cause of fever of unknown origin. Best Pract Res Clin Rheumatol 2016;30:789-801.

[5] Larson EB, Featherstone HJ, Petersdorf RG. Fever of undetermined origin: diagnosis and follow-up of 105 cases, 19701980. Medicine (Baltimore) 1982;61:269-92.

[6] de Kleijn EM, van Lier HJ, van der Meer JW. Fever of unknown origin (FUO). II. Diagnostic procedures in a prospective multicenter study of 167 patients. The Netherlands FUO Study Group. Medicine (Baltimore) 1997;76:401-14.

[7] Gaeta GB, Fusco FM, Nardiello S. Fever of unknown origin: a systematic review of the literature for 1995-2004. Nucl Med Commun 2006;27:205-11.

[8] Vanderschueren S, Knockaert D. Tackling fever and inflammation of unknown origin: the do's and don'ts. Acta Clin Belg 2014;69:412-17. 
[9] Mourad O, Palda V, Detsky AS. A comprehensive evidencebased approach to fever of unknown origin. Arch Intern Med 2003;163:545-51.

[10] Cunha BA. Fever of unknown origin: clinical overview of classic and current concepts. Infect Dis Clin North Am 2007;21:867-915.

[11] Takeda R, Mizooka M, Kobayashi T, Kishikawa N, Yokobayashi K, Kanno K, et al. Key diagnostic features of fever of unknown origin: medical history and physical findings. J Gen Fam Med 2017;18:131-34.

[12] Cunha BA, Lortholary O, Cunha CB. Fever of unknown origin: a clinical approach. Am J Med 2015;128:1138.e11138.e15.

[13] De Kleijn EMHA, Vandenbroucke JP, Van Der Meer JWM, and The Netherlands FUO Study Group. Fever of unknown origin (FUO). I. A prospective multicenter study of 167 patients with FUO, using fixed epidemiologic entry criteria. Medicine (Baltimore) 1997;76:392-400.

[14] Bleeker-Rovers CP, Vos FJ, de Kleijn EM, Mudde AH, Dofferhoff TS, Richter C, et al. A prospective multicenter study on fever of unknown origin. the yield of a structured diagnostic protocol. Medicine (Baltimore) 2007;86:26-38.

[15] Iikuni Y, Okada J, Kondo H, Kashiwazaki S. Current fever of unknown origin 1982-1992. Intern Med 1994;33:67-73.

[16] Cruz Peña LA, Rodríguez Silva H, Pérez Caballero D. Fiebre de origen desconocido: Revisión de 105 pacientes. Rev Cubana Med 1995;34:1-10.

[17] Knockaert DC, Vanneste LJ, Vanneste SB, Bobbaers HJ. Fever of unknown origin in the 1980s. An update of the diagnostic spectrum. Arch Intern Med 1992;152:51-5.

[18] Durack DT, Street AC. Fever of unknown origin-reexamined and redefined. Curr Clin Top Infect Dis 1991;11:35-51.

[19] Marr KA. Actitud ante la fiebre y la sospecha de infección en el huésped inmunodeprimido. En: Goldman L, Schafer AI, editores. Cecil y Goldman. Tratado de Medicina Interna. 24a ed. Barcelona: Elsevier; 2013.p.1778-84.

[20] Hirschmann JV. Fever of unknown origin in adults. Clin Infect Dis 1997;24:291-302.

[21] Hot A, Schmulewitz L, Viard JP, Lortholary O. Fever of unknown origin in HIV/AIDS patients. Infect Dis Clin N Am 2007;21:1013-32.
[22] Knox TA, Wanke C. Gastrointestinal manifestations of HIV and AIDS. In: Goldman L, Schafer AI, editors. Goldman-Cecil Medicine. 25th ed. Philadelphia: Elsevier; 2016. p. 2302-5.

[23] Arnow PM, Flaherty JP. Fever of unknown origin. Lancet 1997;350:575-80.

[24] Cunha BA, Dieguez B, Varantsova A. Lessons learned from splenic infarcts with fever of unknown origin (FUO): culturenegative endocarditis (CNE) or malignancy? Eur J Clin Microbiol Infect Dis 2018;37:995-9.

[25] Cunha BA, Apostolopoulou A, Gian J. Fever of unknown origin (FUO) due to miliary BCG: The diagnostic importance of morning temperature spikes and highly elevated ferritin levels. Heart Lung 2017;46:205-7.

[26] Ellner JJ. Tuberculosis. In: Goldman L, Schafer AI, editors. Goldman-Cecil Medicine. 25th ed. Philadelphia: Elsevier; 2016.p.2030-9.

[27] Burzo ML, Antonelli M, Pecorini G, Favuzzi AMR, Landolfi R, Flex A. Fever of unknown origin and splenomegaly. A case report of blood culture negative endocarditis. Medicine (Baltimore) 2017;96:1-3

[28] Sheon RP, Van Ommen RA. Fever of obscure origin: diagnosis and treatment based on a series of sixty cases. Am J Med 1963;34:486-99.

[29] Abba A, Khalil M. Clinical approach to lymphadenopathy. Ann Nigerian Med 2012;6:11-7.

[30] Sinclair S, Beckman E, Ellman L. Biopsy of enlarged superficial lymph nodes. JAMA 1974;228:602-3.

[31] Watts RA. How to investigate multisystem disease. Best Pract Res Clin Rheumatol 2014;28:831-43.

[32] Bofinger JJ, Schlossberg D. Fever of unknown origin caused by tuberculosis. Infect Dis Clin N Am 2007;21:947-62.

[33] Kim JH, Kim ES, Jun K-I, Jung Hg, Bang JH, Choe PG, et al. Delayed diagnosis of extrapulmonary tuberculosis presenting as fever of unknown origin in an intermediate-burden country. BMC Infect Dis.2018;18:426.

[34] Knockaert DC. Diagnostic strategy for fever of unknown origin in the ultrasonography and computed tomography era. Acta Clin Belg 1992;47:100-16.

[35] Knockaert DC, Vanneste LJ, Bobbaers HJ. Recurrent or episodic fever of unknown origin. Review of 45 cases and survey of the literature. Medicine (Baltimore) 1993;72:184-96. 